\title{
Ground based observation of TNO targets for the Herschel Space Observatory
}

\author{
R. Duffard, J. L. Ortiz, A. Thirouin, P. Santos-Sanz, \\ and N. Morales \\ Instituto de Astrofísica de Andalucía - CSIC, C/ Camino Bajo de Huetor, \\ 50. Granada, 18008, Spain \\ email: duffard@iaa.es
}

\begin{abstract}
We have observed a subset of TNOs that are going to be studied by means of Herschel Space Telescope (HSO). More than 50 objects have been studied astrometrically and 30 with time series photometry. The main conclusion regarding the astrometry is that all the observed HSO targets have ephemerides uncertainties smaller than 5 arcsec, needed for the correct pointing of the space telescope. Concerning the time series analysis of the targets, most of the objects present low amplitude variability. This is an on-going program and more results are expected.
\end{abstract}

Keywords. Trans-neptunian Objects, photometry, astrometry

\section{Introduction}

Transneptunian Objects (TNOs) are believed to represent one of the most primordial populations in the solar system. The TNO population comprises (i) the main Kuiper Belt beyond the orbit of Neptune $(32-50 \mathrm{AU})$, consisting of objects in resonant and non-resonant orbits, (ii) the halo outskirts of "scattered" and "detached" bodies beyond $50 \mathrm{AU}$, and (iii) the Centaurs, which are closer to the Sun and in transition towards the inner solar system where some of them are eventually captured as short-period comets in the Jupiter family (Gladman et al. 2008). About 1200 TNOs have been detected so far and, as detailed hereafter, new studies have started to reveal a richness of orbital and physical properties. These TNOs represent only a few percent of the estimated 30000 TNOs brighter than 24 mag in the visible (Petit et al. 2008).

Herschel Space Observatory (HSO) that was launched this year is set up to make a key contribution to the study of dusty debris disks around other stars. Moreover, the approved Herschel program "TNOs are cool" (Mueller et al. 2009) will obtain thermal data of a set of 140 TNOs. As part of the international group with an approved TNO observational proposal of 372.8 hours in this space Telescope we need ground-based support for some of the selected targets. For the albedo estimations to be made using the HSO measurements, accurate visible photometry is required. Moreover, color information of the TNOs will be used for statistical analysis to study, for instance, correlations between size, albedo, photometric and dynamical parameters, etc. (Santos-Sanz et al. 2009). More than half of the HSO TNO targets require first time photometric measurements or refinement of existing results.

For successful observations with HSO, accurate pointing of the TNOs is fundamental to make best use of the available field of view for jittering and avoidance of bad pixels as well as to stay away from brighter disturbing background sources. This in turn requires a good knowledge of the orbit of the targets to the level of 5 arcsec for the time when HSO will observe the respective TNO. An analysis of the uncertainties in the ephemerides 
of the program targets has revealed that about half of the 140 program objects have prediction errors of 10 arcsec and more (some even several arcmin) in the critical time intervals for the HSO observations, partially because the last astrometry of the objects was obtained years ago and partially because the measured orbital arc is not sampled properly. In order to overcome this compromising situation for the success of the HSO observations, we measured new accurate astrometric positions of more than 50 TNO targets with uncertain ephemerides. The TNO images also allow obtaining photometry of the TNOs for a refinement of the absolute brightness of the targets in reflected sunlight, a result that will be used together with the HSO measurements to determine size and albedo of the objects. It goes without saying that the new astrometry of the HSO targets will contribute to the orbit refinement of the TNOs per se, thus improving the still unsatisfying situation for statistical analysis of the TNO orbits and dynamics. In this work we present some results of the observational campaign we are involved using different ground-based telescopes.

\section{Observations}

Our group at the Instituto de Astrofísica de Andalucía - CSIC started a vast program on lightcurves and astrometry of Kuiper Belt Objects (KBOs) in 2001. Observations were carried out from the $1.5 \mathrm{~m}$ telescope at Sierra Nevada Observatory (OSN - Granada, Spain), from the $2.2 \mathrm{~m}$ telescope at Centro Astronòmico Hispànico Alemàn (CAHA) at Calar Alto Observatory (Almeria, Spain) and from the 2.5m Isaac Newton Telescope (INT), the $2.5 \mathrm{~m}$ Liverpool Telescope (LVPL), the $10.2 \mathrm{~m}$ Gran Telescopio de Canarias (GTC) and the 4.2m William Herschel Telescope (WHT) at El Roque de Los Muchachos Observatory (La Palma, Spain).

Lightcurve observations, aiming to determine the rotational period and amplitude are mainly carried out at the OSN, INT and CAHA $2.2 \mathrm{~m}$. Photometry to determine the TNOs colors were done at the WHT and LVPL and finally, spectroscopic observations in the visible range were done at the GTC.

\section{Results}

All the TNO images taken, not only on purpose astrometric ones, were processed using the USNO-B1 catalogue. The corresponding right ascension and declination coordinates were submitted to the Minor Planet Center and a plot showing the offsets with respect the JPL Horizon system ephemerides is presented for comparison in Fig. 1.

The final time series photometry of each target was inspected for periodicities by means of the Lomb technique (Lomb 1976) as implemented in Press et al. (1992), but we also verified the results by using several other time series analysis techniques (such as PDM), the Harris et al. (1989) method and the CLEAN technique (Foster 1995). Concerning the amplitudes of the short-term variability, we used Fourier fits to the data in order to determine peak to valley amplitudes (full amplitudes). Two examples are shown in Fig. 2 and the complete set is presented in Thirouin et al. (2009). An analysis of the all available lightcurve database in literature is presented in Duffard et al. (2009).

Spectroscopy at GTC in an on-going observational program and the results are currently being analyzed. 


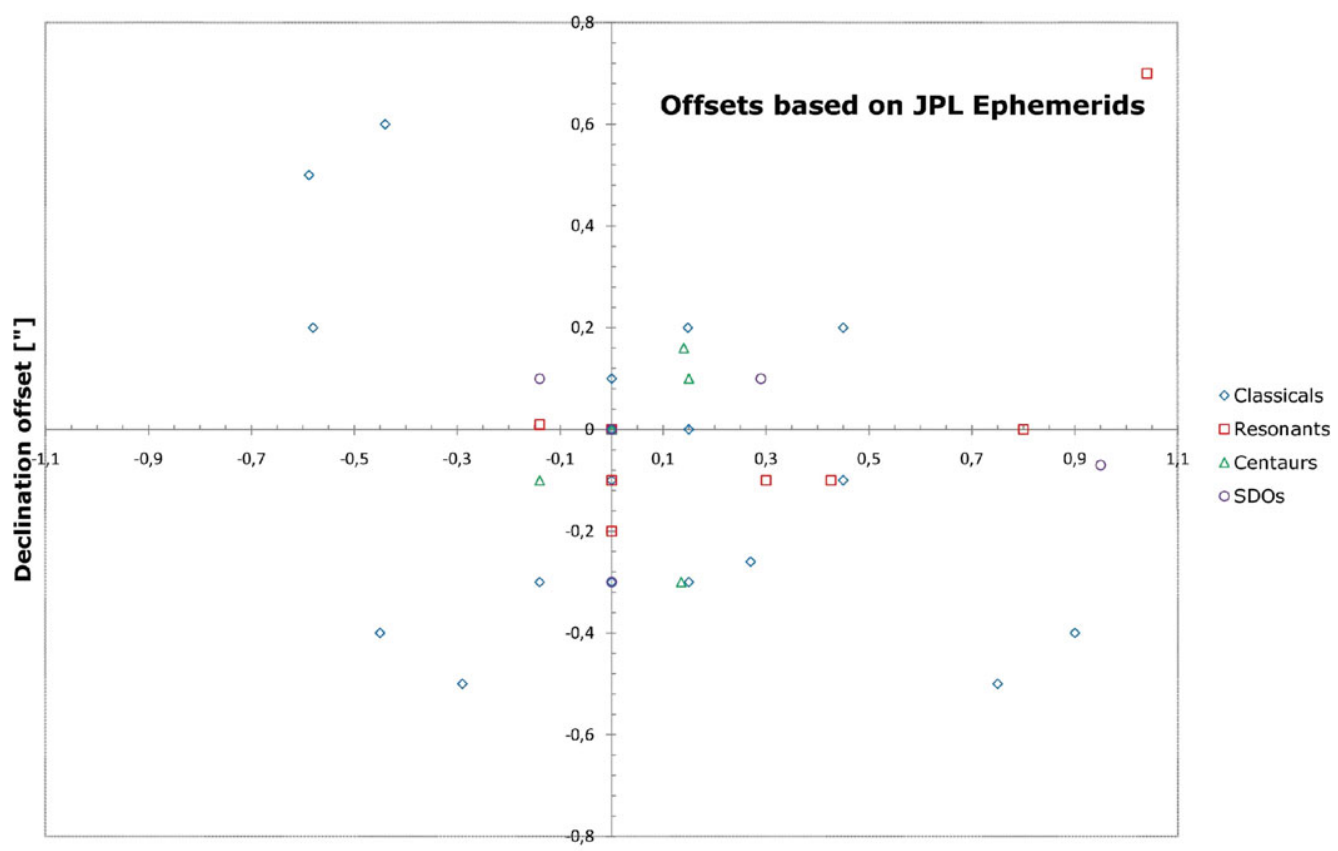

Right ascension offset ["]

Figure 1. Offset in right ascension and declination, compared to the coordinates on JPL Horizon system ephemerides at the time of the observation.
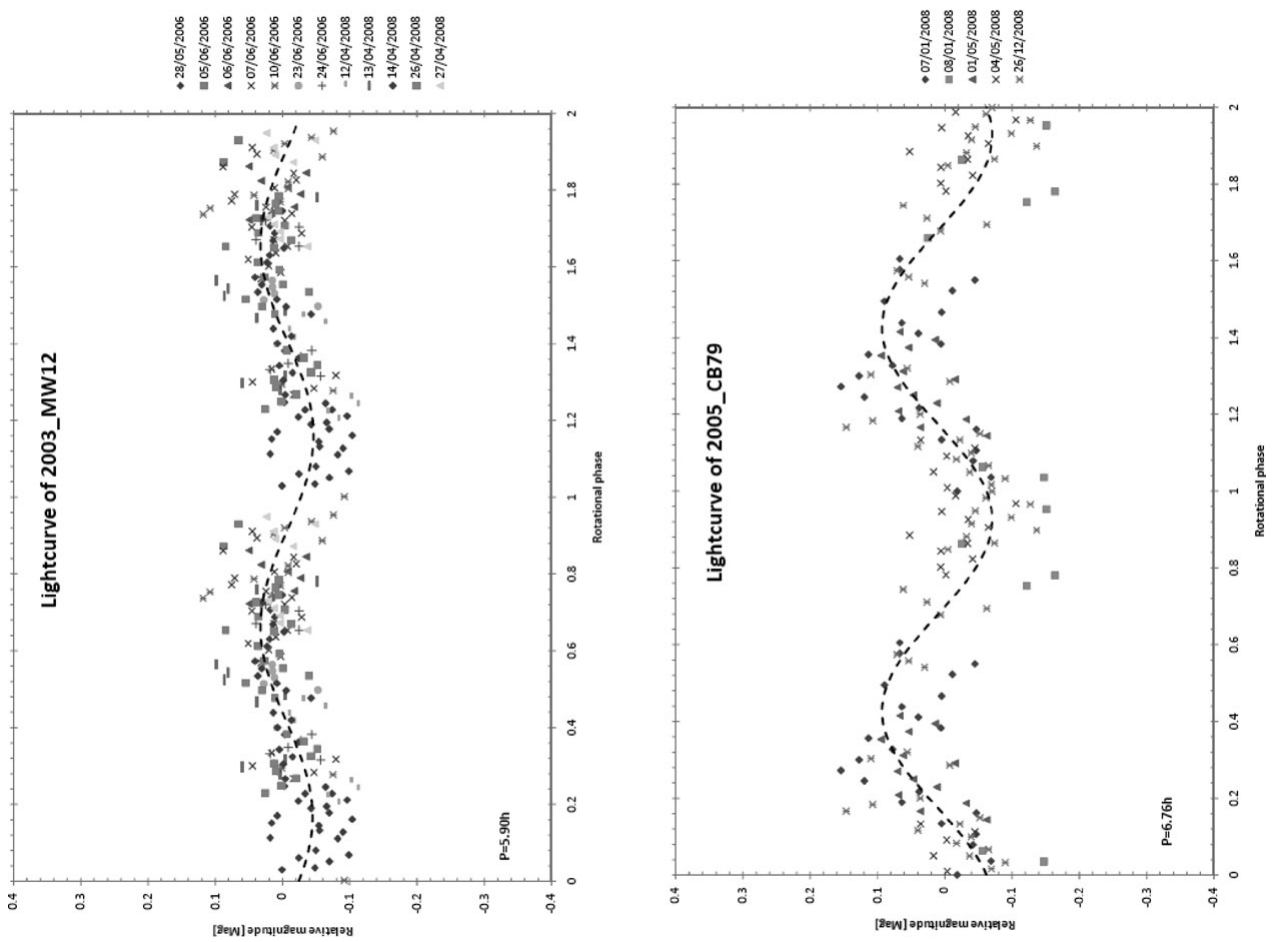

Figure 2. Two examples of lightcurves. A complete set of the newly determined lightcurves is presented in the work by Thirouin et al. (2009). 


\section{Conclusions}

We have observed a subset of TNOs that are going to be studied by means of Herschel Space Telescope. Astrometry observations were aimed to improve the orbits of the selected targets whose positions must be known to high accuracy for Herschel pointing requirements. More than 50 objects have been studied astrometrically. The main conclusion regarding the astrometry is that all the observed HSO targets have ephemerides uncertainties smaller than 5 arcsec, needed for the correct pointing of the space telescope. There are no obvious systematic uncertainties.

Concerning the time series analysis of the targets, most of the objects present low amplitude variability. Low amplitude lightcurves are generally caused by albedo heterogeneity on the surfaces of the bodies (Duffard et al. 2008), although elongated objects seen at certain geometries can give rise to nearly flat lightcurves as well. The physical reason for the presence of many low amplitude rotators in the Kuiper Belt is investigated in more detail in a separate work (Duffard et al. 2009).

\section{References}

Duffard, R., Ortiz, J. L., Santos-Sanz, P., Mora, A., Gutierrez, P. J., Morales, N., \& Guirado, D. $2008, A \mathscr{E} A, 479,877$

Duffard, R., Ortiz, J. L., Thirouin, A., Santos-Sanz, P., \& Morales, N. 2009, A\& A, 505, 1283

Foster, G. 1995, AJ, 109, 1889

Gladman, B., Marsden, B. G., \& Vanlaerhoven, C. 2008, in: M. A. Barucci, H. Boehnhardt, D. P. Cruikshank \& A. Morbidelli (eds.), The Solar System Beyond Neptune, (Tucson: University of Arizona Press), p. 43

Harris, A. W., Young, J. W., \& Bowell, E. 1989, Icarus, 77, 171

Lomb, N. R. 1976, Ap\&SS, 39, 447

Muller, T. G., Lellouch, E., Bohnhardt, H., Stansberry, J., Barucci, A., Crovisier, J., Delsanti, A., Doressoundiram, A., Dotto, E., Duffard, R., \& 23 coauthors 2009, Earth, Moon, and Planets, 105, 209

Petit, J.-M., Kavelaars, J. J., Gladman, B., \& Loredo, T. 2008, in: M. A. Barucci, H. Boehnhardt, D. P. Cruikshank \& A. Morbidelli (eds.), The Solar System Beyond Neptune, (Tucson: University of Arizona Press), p. 71

Press, W. H., Teukolsky, S. A., Vetterling, W. T., \& Flannery, B. P. 1992, Numerical recipes in FORTRAN. The art of scientific computing, (Cambrigde: Cambridge University Press)

Santos-Sanz, P., Ortiz, J. L., Barrera, L., \& Boehnhardt, H. 2009, A\& A, 494, 693

Thirouin, A., Ortiz, J. L., Duffard, R., Santos-Sanz, P., Aceituno, F. J., \& Morales, N. 2009, $A \mathscr{E} A$, submitted 